Military Technical College Kobry El-Kobbah

Cairo, Egypt

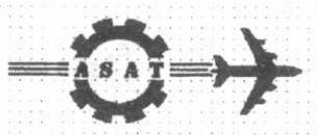

$10^{\text {th }}$ International Conference

On Aerospace Sciences\&

Aviation Technology

\title{
Improving The Positioning Accuracy of High speed Aircraft By using Integrated GPS/INS Navigation System
}

M. Samy Abo el-Soud, M.el-Dakiky, M.el-Khayat

Electrical Engineering Department, Faculty of Engineering At Port Said, Suez Canal University

\section{Abstract:}

Military avionics navigation systems are constantly demanding for exploding system performance and minimizing system size. They require higher accuracy, reliability, and robustness against jamming. This drives the system-integrators towards a generation based upon satellite navigation (GPS) and inertial navigation system (INS).

This paper presents the solution of GPS navigation equations operating in a standalone configuration in a general environment. On the other hand, the solution of INS equations operating in a stand-alone configuration in the same environment.

And finally, the solution of integrated GPS/INS navigation system, which will provide improvements in navigation performance are presented.

\section{Introduction:}

GPS is expected to operate in a stand-alone configuration in a general environment. Users determine their position by GPS receiver by measuring the range between their antenna and four satellites fig. (1). The measured range by the GPS receiver is referred to as "Pseudorange". Mathematically, Pseudorange may be expressed by the equation [1]:

where

$$
\mathbf{R}_{\mathbf{p}}=\mathbf{R}_{\mathbf{a}}+\mathbf{C \Delta} \mathbf{T}_{\mathbf{b}}
$$

$R_{p}$ is the measured Pseudoranges.

$R_{a}$ is the actual or true range.

$\mathrm{C}$ is the speed of light.

$\Delta T_{b}$ is the receiver's clock bias or clock offset.

Since the GPS receiver calculates its position in an Earth Fixed Earth Centered (EFEC) Cartesian-coordinate system, the equation above can be written as follow:

$$
R_{p i}=\sqrt{\left[\left(X-X_{i}\right)^{2}+\left(y-y_{i}\right)^{2}+\left(Z-Z_{i}\right)^{2}\right]}+C \Delta T_{b}
$$

where, $x_{i}, y_{i}, z_{i}$ are position of the $i$-th satellite $(i=1,2,3,4), x, y, z$ are user position (unknown).

The resulting four equations are called "Navigation Equations", and their solution requires measurements of the four pseudoranges to four different satellites. Navigation equations solution using direct method technique and Kalman filter principle are discussed and the differences between the errors obtained from each method are discussed and plotted. 


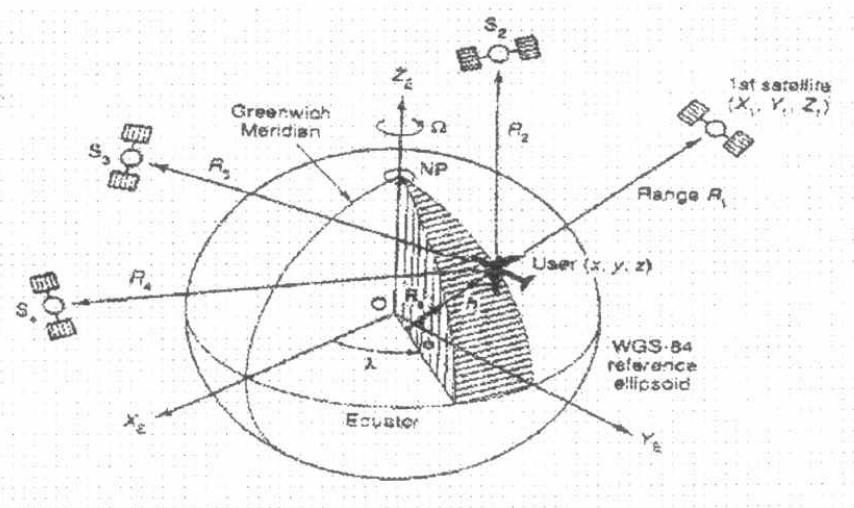

Fig. (1) GPS measured ranges in EFEC-coordinate frame

On the other hand, INS provides high accuracy in short trip navigation but it suffers from error accumulations with time. The estimate of INS error parameters allows in-flight alignment of INS and INS navigation with substationally smaller errors than that could be achieved with INS stand-alone navigator. INS is capable to provide accurate "aiding" data on short-term vehicle dynamics to GPS receiver. By utilizing those aiding signals to effectively reduce the dynamics of the signals to be tracked, the GPS receiver can maintain relatively low tracking bandwidths even in high dynamics environment. When noise-to-signal ratios become so high that tracking of GPS signals is impossible, the INS is capable of navigating independently. When GPS signal conditions improve sufficiently to allow tracking, the INS provides data on initial position, velocity, and acceleration for use in reacquiring the GPS codes and carrier quickly. The INS also provides data for use in adapting the tracking loop parameters to varying conditions of signal dynamics and signal-to-noise ratios, there by improving the ability of the tracking loops to acquire and maintain lock on the GPS signal.

In this paper, we investigate the integration of GPS/INS system to improve the positioning accuracy of a high-speed aircraft.

The rest of the paper is organized as follows: Section $I I$ presents an overview of the estimated aircraft flight parameters; section III presents the solution of GPS navigation equations; section IV presents the solution of INS navigation equations. Solution using GPS/INS integrated navigation system is introduced in section V, with conclusions given in section VI.

\section{Overview of the estimated Aircraft flight parameters:}

The trajectory that the Aircraft should follow during the flight period is assumed to be piece-wise linear path starting from Cairo $\left(30^{\circ}\right.$ lat; $31.3^{\circ}$ long $)$ and ending after $20 \mathrm{~min}$.

Aircraft dynamicai model is assumed to be Position-Velocity-Acceleration (PVA) model.

It is assumed that the equations of motion of the aircraft are given by:

$$
\text { and } \begin{aligned}
d_{k} & =d_{k-1}+V_{k} T, \\
V_{k} & =V_{k-1}+A_{k} T, \\
A_{k} & =\alpha A_{k-1}+w_{k}
\end{aligned}
$$


where

$d_{k}, V_{k}, A_{k}$ are the distance, velocity, and acceleration of the aircraft at $t_{k}$; respectively.

$T$ is the integration step period.

$w_{k}$ is the zero-mean white -noise process of variant $\sigma_{w}^{2}$.

$\alpha$ is the acceleration correlation coefficient, which is given by [2]:

$$
\boldsymbol{\alpha}=1-\frac{T}{M}, \text { for } T \leq M
$$

and

$$
\alpha=0 \quad \text { for } \mathbf{T}>\mathbf{M}
$$

where

$M$ is the average maneuvering duration.

\section{Solution using GPS Navigation equations:}

(1) Using Direct method: Let $\left(X_{n}, Y_{n}, Z_{n}, T_{n}\right)$ be the nominal point or the point of linearization of $(X, Y, Z, T)$ variables, $(\Delta X, \Delta Y, \Delta Z, \Delta T)$ be corrections to the nominal values, $\left(R_{n i}\right)$ be the nominal pseudorange measurement to the $i$-th satellite, and $\left(\Delta R_{i}\right)$ be the residual (difference) between actual and nominal range measurements. Thus, the following incremental relationships are obtained $[1,2,3]$ :

$$
\mathbf{X}=\mathbf{X}_{\mathbf{n}}+\Delta \mathbf{X}, \mathbf{Y}=\mathbf{Y}_{\mathbf{n}}+\Delta \mathbf{Y}, \mathbf{Z}=\mathbf{Z}_{\mathbf{n}}+\Delta \mathbf{Z}, \mathbf{T}=\mathbf{T}_{\mathbf{n}}+\Delta \mathbf{T}, \mathbf{R}_{\mathrm{pi}}=\mathbf{R}_{\mathrm{ni}}+\Delta \mathbf{R}_{\mathrm{pi}}
$$

Using Taylor's Series to linearize the navigation equations we get:

$$
\Delta R_{i}=R_{p i}-R_{n i}=\left[\left(\frac{\delta R_{p i}}{\delta X}\right)_{n} \Delta X+\left(\frac{\delta R_{p i}}{\delta Y}\right)_{n} \Delta Y+\left(\frac{\delta R_{P i}}{\delta Z}\right) \Delta Z+\left(\frac{\delta R_{P i}}{\delta T}\right)_{n} \Delta T\right]
$$

where

$\left(\frac{\delta R_{p i}}{\delta X}\right)_{n},\left(\frac{\delta R_{p i}}{\delta Y}\right)_{n},\left(\frac{\delta R_{P i}}{\delta Z}\right),\left(\frac{\delta R_{P i}}{\delta T}\right)_{n}$ are the pseudorange derivatives calculated at the nominal point $\left(\mathbf{X}_{n}, \mathbf{Y}_{\mathbf{n}}, \mathbf{Z}_{\mathrm{n}}, \mathbf{T}_{\mathbf{n}}\right)$.

Equation (4) gives us four linearized equations, which can be put in the matrix form as follows:

$$
\left[\begin{array}{l}
\Delta R_{p 1} \\
\Delta R_{p 2} \\
\Delta R_{p 3} \\
\Delta R_{p 4}
\end{array}\right]=\left[\begin{array}{llll}
\left(\delta R_{p 1} / \delta X\right)_{n} & \left(\delta R_{p 1} / \delta Y\right)_{n} & \left(\delta R_{p 1} / \delta Z\right)_{n} & \left(\delta R_{p 1} / \delta T\right)_{n} \\
\left(\delta R_{p 2} / \delta X\right)_{n} & \left(\delta R_{p 2} / \delta Y\right)_{n} & \left(\delta R_{p 2} / \delta Z\right)_{n} & \left(\delta R_{p 2} / \delta T\right)_{n} \\
\left(\delta R_{p 3} / \delta X\right)_{n} & \left(\delta R_{p 3} / \delta Y\right)_{n} & \left(\delta R_{p 3} / \delta Z\right)_{n} & \left(\delta R_{p 3} / \delta T\right)_{n} \\
\left(\delta R_{p 4} / \delta X\right)_{n} & \left(\delta R_{p 4} / \delta Y\right)_{n} & \left(\delta R_{p 4} / \delta Z\right)_{n} & \left(\delta R_{p 4} / \delta T\right)_{n}
\end{array}\right]\left[\begin{array}{c}
\Delta X \\
\Delta Y \\
\Delta Z \\
\Delta T
\end{array}\right]
$$

or,

$$
\mathbf{R}=\mathbf{H X}
$$

where

$$
R=\left[\begin{array}{llll}
\Delta R_{1} & \Delta R_{2} & \Delta R_{3} & \Delta R_{4}
\end{array}\right]^{T}
$$




$$
H=\left(\begin{array}{llll}
\left(\delta R_{p 1} / \delta X\right)_{n} & \left(\delta R_{p 1} / \delta Y\right)_{n} & \left(\delta R_{p 1} / \delta Z\right)_{n} & \left(\delta R_{p 1} / \delta T\right)_{n} \\
\left(\delta R_{p 2} / \delta X\right)_{n} & \left(\delta R_{p 2} / \delta Y\right)_{n} & \left(\delta R_{p 2} / \delta Z\right)_{n} & \left(\delta R_{p 2} / \delta T\right)_{n} \\
\left(\delta R_{p 3} / \delta X\right)_{n} & \left(\delta R_{p 3} / \delta Y\right)_{n} & \left(\delta R_{p 3} / \delta Z\right)_{n} & \left(\delta R_{p 3} / \delta T\right)_{n} \\
\left(\delta R_{p 4} / \delta X\right)_{n} & \left(\delta R_{p 4} / \delta Y\right)_{n} & \left(\delta R_{p 4} / \delta Z\right)_{n} & \left(\delta R_{p 4} / \delta T\right)_{n}
\end{array}\right)
$$

and

$$
\mathbf{X}=[\mathbf{\Delta X} \Delta \mathbf{Y} \Delta \mathbf{Z} \Delta \mathbf{T}]^{\mathrm{T}}
$$

Taking the inverse of the matrix $H$ then we can solve the position error and clock bias $\mathrm{X}=\mathrm{H}^{-1} \mathbf{R}$

By adding the calculated position error and clock bias to the values, we get the on time position and time.

(2) Using Kalman Filter: the Aircraft dynamics can be represented in matrix form as $[4,5,6]$ :

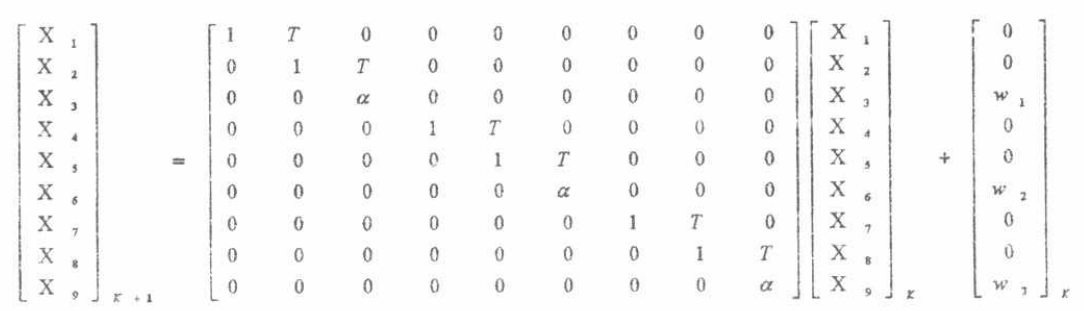

where

$X_{1}=$ EFEC $x$ - axis position, $\quad X_{2}=$ EFEC $x$ - axis velocity.

$X_{3}=$ EFEC $x$ - axis acceleration, $X_{4}=$ EFEC $y$ - axis position.

$\mathbf{X}_{5}=$ EFEC $\mathbf{y}$ - axis velocity, $\quad \mathbf{X}_{6}=$ EFEC $y$ - axis acceleration.

$\mathrm{X}_{7}=$ EFEC $\mathrm{z}$ - axis position, $\quad \mathrm{X}_{8}=$ EFEC $\mathrm{z}$ - axis velocity

$\mathrm{X}_{9}=$ EFEC $\mathrm{z}$ - axis acceleration.

$w_{1}, w_{2}, w_{3}$ are white- Gaussian noise of zero mean and variances of $\sigma^{2}{ }_{w 1}, \sigma^{2}{ }_{w 2}, \sigma^{2}{ }_{w 3}$ The state error Covariance matrix is given by:

$$
Q=E\left\{w w^{T}\right\}=\left[\begin{array}{ccccccccc}
0 & 0 & 0 & 0 & 0 & 0 & 0 & 0 & 0 \\
0 & 0 & 0 & 0 & 0 & 0 & 0 & 0 & 0 \\
0 & 0 & \sigma^{2}{ }_{w 1} & 0 & 0 & 0 & 0 & 0 & 0 \\
0 & 0 & 0 & 0 & 0 & 0 & 0 & 0 & 0 \\
0 & 0 & 0 & 0 & 0 & 0 & 0 & 0 & 0 \\
0 & 0 & 0 & 0 & 0 & \sigma^{2}{ }_{w 2} & 0 & 0 & 0 \\
0 & 0 & 0 & 0 & 0 & 0 & 0 & 0 & 0 \\
0 & 0 & 0 & 0 & 0 & 0 & 0 & 0 & 0 \\
0 & 0 & 0 & 0 & 0 & 0 & 0 & 0 & \sigma^{2}{ }_{w 3}
\end{array}\right]
$$


GPS system Pseudorange measurement noise is assumed to be white Gaussian noise of standard deviation $[100 \mathrm{~m}]$.

Hence, the measurements model of GPS receiver can be in the form:

$\left[\begin{array}{c}R_{p 1}-R_{p n 1} \\ R_{p 2}-R_{p n 2} \\ R_{p 3}-R_{p n 3}\end{array}\right]_{K}=\left[\begin{array}{lllllllll}h_{x}^{1} & 0 & 0 & h_{y}^{1} & 0 & 0 & h_{z}^{1} & 0 & 0 \\ h_{x}^{2} & 0 & 0 & h_{y}^{2} & 0 & 0 & h_{z}^{2} & 0 & 0 \\ h_{x}^{3} & 0 & 0 & h_{y}^{3} & 0 & 0 & h_{z}^{3} & 0 & 0\end{array}\right]_{n}\left[\begin{array}{l}\mathrm{X}_{1} \\ \mathrm{X}_{2} \\ \mathrm{X}_{3} \\ \mathrm{X}_{4} \\ \mathrm{X}_{5} \\ \mathrm{X}_{6} \\ \mathrm{X}_{7} \\ \mathrm{X}_{8} \\ \mathrm{X}_{9}\end{array}\right]+\left[\begin{array}{c}\Gamma_{1} \\ \Gamma_{2} \\ \Gamma_{3}\end{array}\right]$

where

$\left[\begin{array}{lll}\Gamma_{1} & \Gamma_{2} & \Gamma_{3}\end{array}\right]$ is the white-Gaussian measurement noise.

In simulation algorithm, the Aircraft flies over Cairo at One O'clock AM and it is required to follow the trajectory described above. The receiver used is assumed to be multichannel receiver, operates on $\mathrm{C} / \mathrm{A}$ code only. The required ephemeris data are obtained from a stored file containing all ephemeris data for the best four satellites (have min. GDOP), which are visible at this time over Cairo.

Simulation program is executed 10-times and the average values of errors from each technique are plotted as shown in figs. (2) and (3).

Fig. (2-a) through (2-c) illustrates the EFEC-Cartesian average position errors obtained from each method. Fig. (3) illustrates the polar average position errors obtained from each method. It is clear from the plotting that, the Kalman filter method is better than direct method table (1).

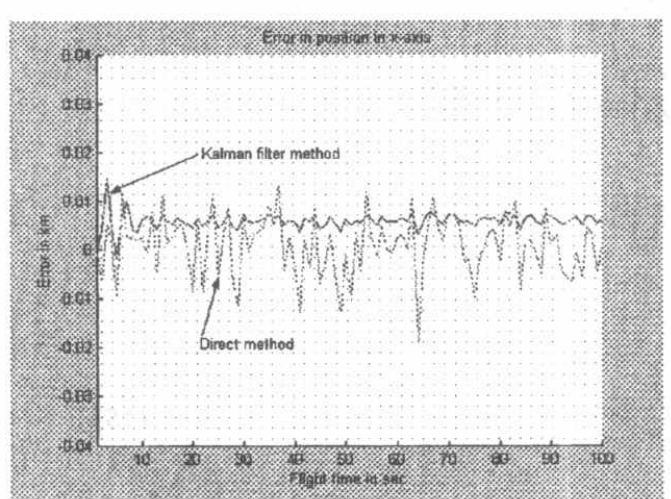

Fig. (2-a) Error in position in $\mathrm{X}$-axis

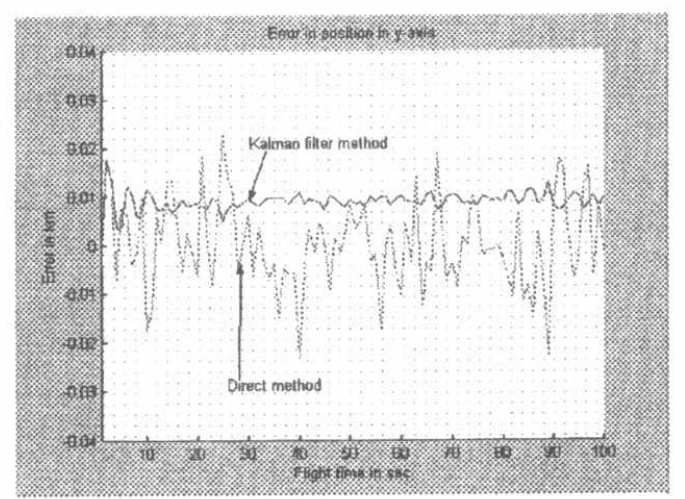

Fig. (2-b) Error in position in Y-axis 


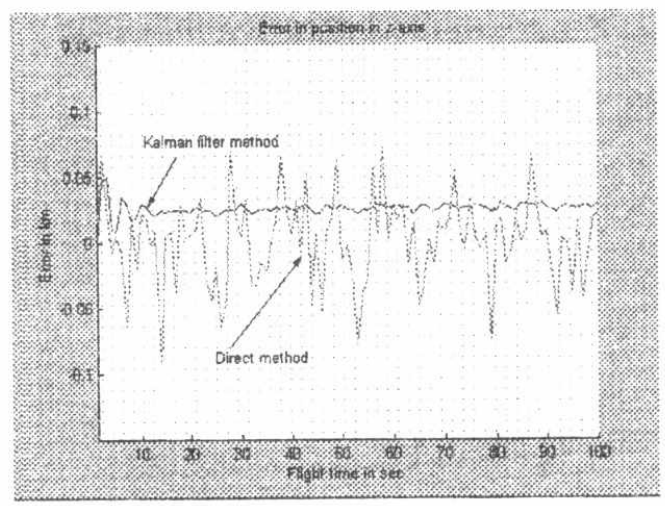

Fig. (2-c) Error in position in $\mathrm{Z}$-axis

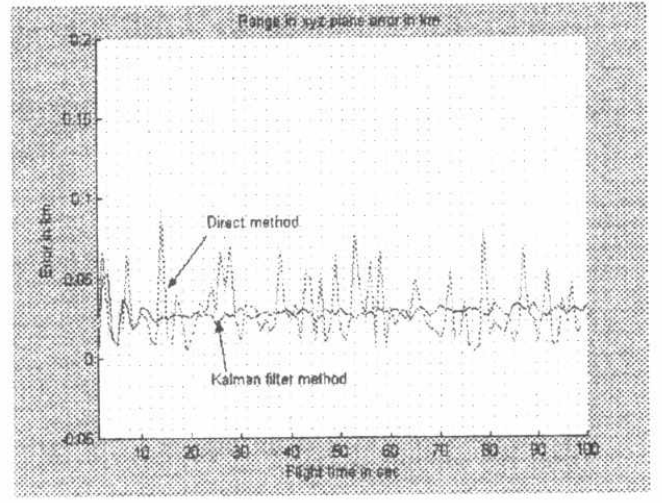

Fig. (3) Error in Range in XYZ-plane

IV. Solution Using INS navigation equations:

The INS outputs are attitude, velocity and position. Attitude is computed directly in case of gimbaled system, and calculated from the solution of the direction cosine matrix differential equations in strapdown system. The strapdown configuration has several advantages; elimination of gimbals leads to lower system costs, smaller mechanical and electrical form vector, and increased reliability,

Position and velocity are calculated by solving the navigation equations that describes the motion of the vehicle with respect to a certain reference frame. The most famous reference frame is local-geographical (navigation) frame because it is simple and used for long distance navigation.

The general INS navigation equations used for the geographical frame (assuming the case of north-east-down frame, spherical earth model and constant Earth's gravitational field) are given in the vector form by [7,8]:

$$
\begin{aligned}
\dot{v_{N}} & =f_{N}-v_{E}(2 \Omega+\dot{\lambda}) \sin L+v_{D} \dot{L}+\zeta_{g} \\
& =f_{N}-2 \Omega v_{E} \sin L+\frac{\left(v_{N} v_{D}-v_{E}^{2} \tan L\right)}{\left(R_{o}+h\right)}+\zeta_{g} \\
\dot{v_{E}} & =f_{E}+v_{N}(2 \Omega+\dot{\lambda}) \sin L+v_{D}(2 \Omega+\dot{\lambda}) \cos L-\eta g \\
& =f_{E}-2 \Omega\left(v_{N} \sin L+v_{D} \cos L\right)+\left(\frac{v_{B}}{R_{o}+h}\right)\left(v_{D}+v_{N} \tan L\right)-\eta g \\
\dot{v_{D}} & =f_{D}-v_{E}(2 \Omega+\dot{\lambda}) \cos L-v_{N} \dot{L}+g \\
& =f_{D}-2 \Omega v_{E} \cos L-\left(\frac{v_{E}^{2}-v_{N}^{2}}{R_{o}+h}\right)+g
\end{aligned}
$$

where

$L$ is the latitude of the vehicle.

$V_{N}, V_{E}, V_{D}$ are north, east, and down velocity components; respectively. 
$f_{N}, f_{E}, f_{D}$ are north, east, and down acceleration components due to applied specific force; respectively.

$h$ is the height of the vehicle over the earth.

$\zeta$ and $\eta$ represent angular deflections in the direction of the local gravity vector with respect to the local vertical due to gravity anomalies.

Latitude, longitude and height above the surface of the earth are given by $[7,8]$ :

$$
\begin{aligned}
L^{*} & =\frac{v_{N}}{\left(R_{o}+h\right)} \\
\lambda^{\bullet} & =\frac{v_{E} \sec L}{\left(R_{o}+h\right)} \\
h^{\bullet} & =-v_{D}
\end{aligned}
$$

It is assumed, in the equations given above, that the earth is perfectly spherical in shape, additionally, it is assumed that there is no variation in the earth's gravitational field with changes in the position of the navigation system on the earth or its height above the surface of the earth.

In accordance with this model (WGS-84) the following parameters are defined [8]:

Length of the semi-major axis

Length of the semi-minor axis

$$
\begin{aligned}
R & =6378137 \mathrm{~m} \\
r & =R(1-f)=6356752.3142 \mathrm{~m}
\end{aligned}
$$$$
\mathrm{f}=(\mathrm{R}-\mathrm{r}) / \mathrm{R}=1 / 298.257223563
$$

Flattening of the ellipsoid

$\mathrm{e}=[\mathrm{f}(2-\mathrm{f})]^{1 / 2}=0.0818191908426$

$\begin{array}{ll}\text { Major eccentricity of the ellipsoid } e=[f(2-f)]^{1 / 2}=0.0818191908 \\ \text { Earth's rate } & \Omega=7.292115 \times 10^{-5} \mathrm{rad} / \mathrm{sec}\end{array}$

By modeling the earth in accordance with the reference ellipsoid defined above, the rates of change of latitude and longitude may be expressed in terms of a meridian radius of curvature $\left(\mathbf{R}_{\mathrm{N}}\right)$ and a transverse radius of curvature $\left(\mathbf{R}_{\mathrm{E}}\right)$ as follows:

$$
\begin{aligned}
R_{N} & =\frac{R\left(1-e^{2}\right)}{\left(1-e^{2} \operatorname{Sin}^{2} L\right)^{3 / 2}} \\
R_{E} & =\frac{R}{\left(1-e^{2} \operatorname{Sin}^{2} L\right)^{1 / 2}}
\end{aligned}
$$

The mean radius of curvature used in the earlier equations is $R_{0}=\left(\mathbf{R}_{\mathrm{E}^{*}} \mathbf{R}_{N}\right)^{1 / 2}$. Similarly, the transport rate now takes the following form:

$$
\boldsymbol{\omega}_{\mathrm{en}} \mathbf{n}=\left[\frac{\mathrm{V}_{\mathrm{E}}}{R_{E}+h}-\frac{V_{N}}{R_{N}+h}-\frac{V_{E} \tan L}{R_{N}+h}\right]^{T}
$$

The deflection of the local gravity vector from the vertical may be expressed as angular deviations about the north and east axes of local geographic frame as follows [8]:

$$
\mathbf{g}=[\zeta g,-\eta \mathbf{g}, \mathbf{g}]
$$

where $\zeta$ is the meridian deflection and $\eta$ is the deflection perpendicular to the meridian. The resulting deviation of the vertical over the surface of the earth varies by up to 30 arc seconds.

Exact knowledge of the magnitude of gravity is also vital for accurate navigation. The following expressions for the variation of the magnitude of the gravity vector with latitude at sea level $(h=0)$ and its rate of change with height above ground [15]: 


$$
\begin{aligned}
& g(0)=9.780318\left(1+5.3024 .10^{-3} \sin ^{2} \mathrm{~L}-5.9 .10^{-6} \sin ^{2} 2 \mathrm{~L}\right) \mathrm{m} / \mathrm{sec}^{2} \\
& \frac{\mathrm{d}}{\mathrm{dh}} \mathrm{g}(0)=-0.0000030877\left(1-1.39 .10-3 \sin ^{2} \mathrm{~L}\right) \mathrm{m} / \mathrm{sec}^{2} / \mathrm{m}
\end{aligned}
$$

It is sufficient to assume that the variation of gravity with altitude is as follows [8]:

$$
g(h)=g(0) /(1+h / R o)^{2}
$$

The solution of the above navigation equations gives the position and velocity of the user. The difference between the solution of the above navigation equation in gimbaled system and in strapdown system is that for gimbaled system, the measured specific force accelerations sensed by accelerometers are directly applied to the navigation equation, while in strapdown system, these measurements must be resolved into the navigation frame using the measured attitude before applying to the navigation equation (i.e. calculated from the solution of the direction cosine matrix differential equations). In simulation, the pendulous accelerometer is assumed to be used which has a random error term modeled as zero-mean white Gaussian noise of typical value of variance $\left(\sigma_{a}^{2}=\left(1 \mathrm{~m} / \mathrm{sec}^{2}\right)^{2}\right)[8]$, scale factor error given by the typical value $(S=0.05 \%)$, and fixed bias $(B=0.01 \mathrm{~g})$, while the other terms are not modeled as they are considered very small values.

Scenario: the used INS mechanization is strapdown local-geographical system, with the navigation axes mounted as: North, East, and Down (i.e. NED frame) assuming the ellipsoid model of the earth (WGS-84).

The program starts by reading the acceleration information from the flight path file (which are in EFEC-frame) and transforming it to the NED-coordinate frame then adding the noises to these transformed accelerations. These noisy accelerations are considered the input to the navigation equations (14), (15) and (16). The RungeKutta4 numerical integration method is used to solve these navigation equations, taking the initial position in Cairo location, to get the velocity and position measured in navigation frame.

The position and velocity errors in EFEC-frame are calculated and plotted.

It is found from the plots that the errors are accumulated and increasing with time as shown in fig. (4).

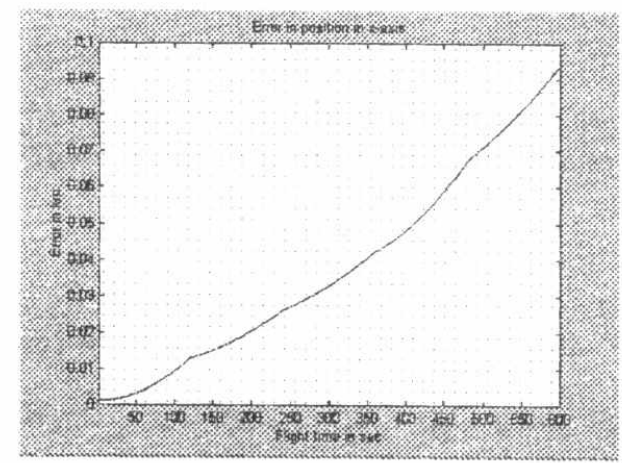

Fig. (4-a) Error in position in X-axis

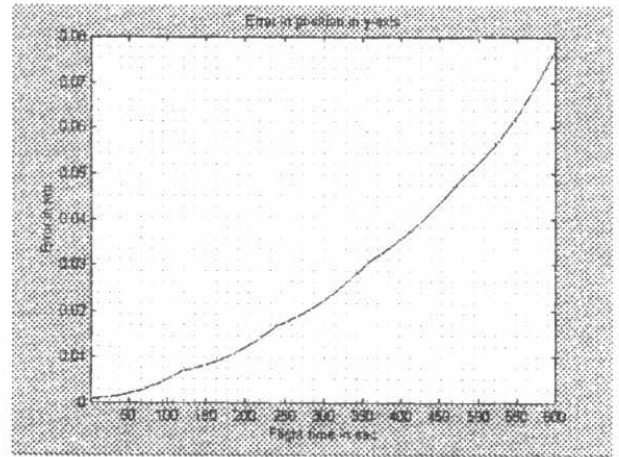

Fig. (4-c) Error in position in Y-axis 


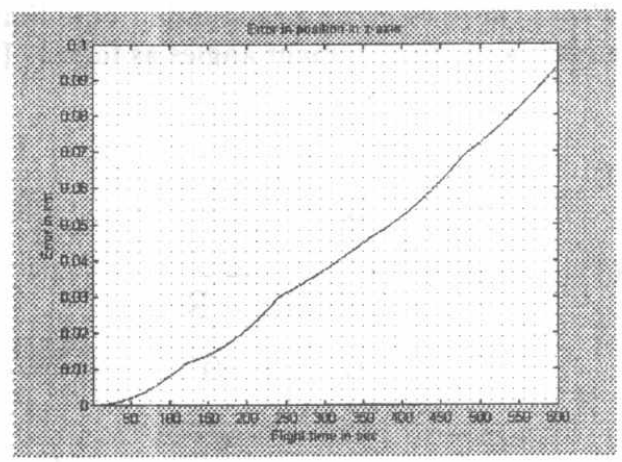

Fig. (4-b) Error in position in Z-axis

\section{Integrated GPS/INS system:}

The global positioning system (GPS) offers an absolute positioning accuracy of 15-100 m. Inertial navigation complements GPS in that it provides relative positioning and is totally self-contained. These two positioning sensors are ideally suited for system integration for although there is not necessarily an improvement in accuracy, the integration of GPS with inertial navigation systems INS does enable an increase in system performance.

Two basic architectures are available to the designer for integrated GPS/INS .The first is named cascaded architectures (Loose) and the second is named fully integrated architecture (Tight). Cascaded architecture has many advantages:

1) The ability to integrate any GPS receiver and any INS.

2) GPS and INS Kalman filters are smaller than the filter required for fully integrated architecture, we use in this simulation the cascaded closed-loop type as shown in fig. (5)[9].

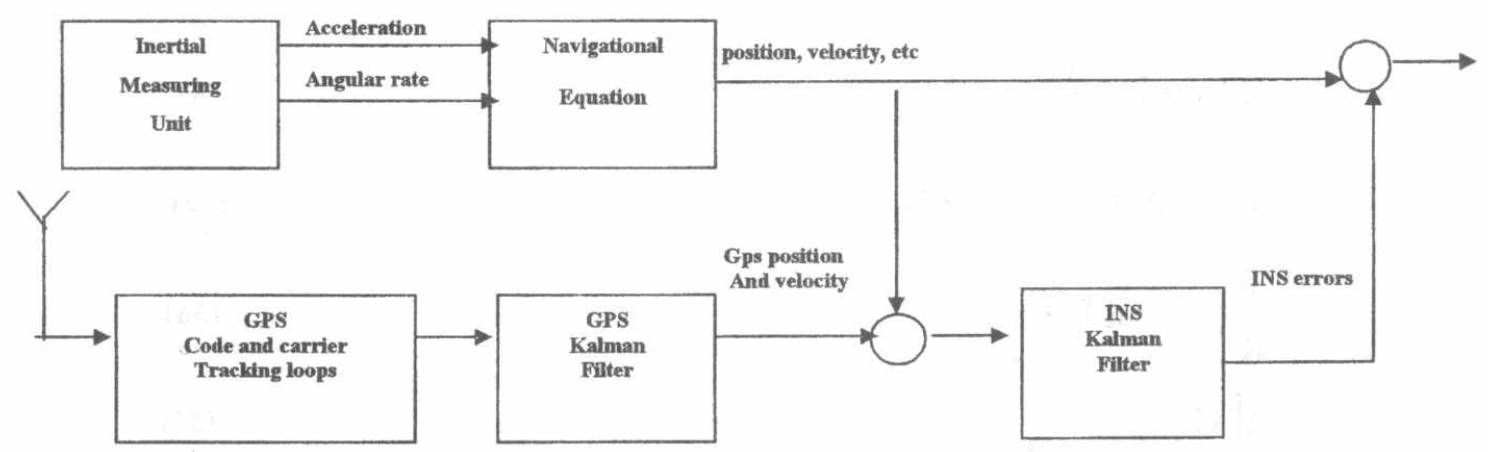

Fig. (5) Cascaded Kalman filter for GPS/INS integration 
System Equation: From the INS error propagation equation discussed before, we take the states of velocity and misalignment angles as follows [8]:

$$
\left[\begin{array}{c}
\delta \dot{\alpha} \\
\delta \dot{\beta} \\
\dot{\delta} \\
\delta \mathrm{v}_{\mathrm{N}}^{\cdot} \\
\delta \mathrm{v}_{\mathrm{B}}^{\cdot} \\
\delta \mathrm{v}_{\mathrm{D}}^{\cdot}
\end{array}\right]=\left[\begin{array}{cccccc}
0 & \mathrm{C}_{2} & \frac{\mathrm{v}_{\mathrm{N}}}{\mathrm{R}} & 0 & -\frac{1}{\mathrm{R}} & 0 \\
\mathrm{C}_{1} & 0 & \mathrm{C}_{4} & -\frac{1}{\mathrm{R}} & 0 & 0 \\
-\frac{\mathrm{v}_{\mathrm{N}}}{\mathrm{R}} & \mathrm{C}_{3} & 0 & 0 & -\frac{\tan \mathrm{L}}{\mathrm{R}} & 0 \\
0 & -\mathrm{f}_{\mathrm{D}} & \mathrm{f}_{\mathrm{B}} & \frac{\mathrm{v}_{\mathrm{D}}}{\mathrm{R}} & \mathrm{C}_{6} & \frac{\mathrm{v}_{\mathrm{N}}}{\mathrm{R}} \\
\mathrm{f}_{\mathrm{D}} & 0 & -\mathrm{f}_{\mathrm{N}} & \mathrm{C}_{5} & \mathrm{C}_{7} & \mathrm{C}_{9} \\
-\mathrm{f}_{\mathrm{E}} & \mathrm{f}_{\mathrm{N}} & 0 & -\frac{2 \mathrm{v}_{\mathrm{N}}}{\mathrm{R}} & \mathrm{C}_{8} & 0
\end{array}\right]\left[\begin{array}{l}
\delta \alpha \\
\delta \beta \\
\delta \gamma \\
\delta \mathrm{v}_{\mathrm{N}} \\
\delta \mathrm{v}_{\mathrm{E}} \\
\delta \mathrm{v}_{\mathrm{D}}
\end{array}\right]+\left[\begin{array}{l}
\mathrm{v}_{\mathrm{gz}} \\
\mathrm{v}_{\mathrm{g}} \\
\mathrm{v}_{\mathrm{gz}} \\
\mathrm{v}_{\mathrm{ax}} \\
\mathrm{v}_{\mathrm{ay}} \\
v_{\mathrm{az}}
\end{array}\right]
$$

where

$$
\begin{aligned}
& C_{1}=\Omega \sin L+\frac{v_{E}}{R} \tan L \\
& C_{2}=-\sin L-\frac{v_{E}}{R} \tan L \\
& C_{3}=-\Omega \cos L-\frac{v_{E}}{R} \\
& C_{4}=\Omega \cos L+\frac{v_{E}}{R} \\
& C_{5}=2 \Omega \sin L+\frac{v_{E}}{R} \tan L \\
& C_{6}=-2\left(\Omega \sin L+\frac{v_{E}}{R} \tan L\right) \\
& C_{7}=\frac{1}{R}\left(v_{N} \tan L+v_{D}\right) \\
& C_{8}=-2\left(\Omega \cos L-\frac{v_{E}}{R}\right) \\
& C_{9}=2 \Omega \cos L+\frac{v_{E}}{R} \\
& \text { R }
\end{aligned}
$$


$v_{g x}, v_{g y}, v_{g z}$ is the gyros noise components.

$v_{a x}, v_{a y}, v_{a z}$ is the accelerometers noise components.

L is the latitude of the vehicle.

$\alpha, \beta, \gamma \quad$ are misalignment angles.

The measurements equations for the Kalman filter will take the form:

$$
\left[\begin{array}{c}
\mathrm{V}_{\mathrm{EG}}-\mathrm{V}_{\mathrm{EI}} \\
\mathrm{V}_{\mathrm{NG}}-\mathrm{V}_{\mathrm{NI}} \\
\mathrm{V}_{\mathrm{DG}}-\mathrm{V}_{\mathrm{DI}}
\end{array}\right]=\left[\begin{array}{llllll}
0 & 0 & 0 & 1 & 0 & 0 \\
0 & 0 & 0 & 0 & 1 & 0 \\
0 & 0 & 0 & 0 & 0 & 1
\end{array}\right]\left[\begin{array}{c}
\alpha \\
\beta \\
\gamma \\
\delta \mathrm{V}_{\mathrm{E}} \\
\delta \mathrm{V}_{\mathrm{N}} \\
\delta \mathrm{V}_{\mathrm{D}}
\end{array}\right]+\left[\begin{array}{c}
\mathrm{v}_{\mathrm{E}} \\
\mathrm{V}_{\mathrm{N}} \\
\mathrm{V}_{\mathrm{D}}
\end{array}\right]
$$

where

$V_{E G}, V_{N G}, V_{D G}$ are the east, north, and up GPS velocity components; respectively.

$\mathrm{V}_{\mathrm{EI}}, \mathrm{V}_{\mathrm{NI}}, \mathrm{V}_{\mathrm{DI}}$ are the east, north, and up INS velocity components ; respectively.

$V_{E}, V_{N}, V_{D}$ are the GPS measurement velocity noise errors; respectively.

In the integrated GPS/INS simulation program, the cascaded Kalman filter architecture is used. The results from the two previous simulation programs of the INS stand-alone and GPS stand-alone are subtracted from each other and the difference is considered the measurements for the INS Kalman filter, which operates on errors only. The same trajectory and a/c dynamic model that were used in GPS simulation, are used here except that the state vector is representing the misalignment angles and velocity errors.

The simulation program is executed $\mathbf{1 0}$ times and the average of the errors are plotted in figs. (6)\&(7).

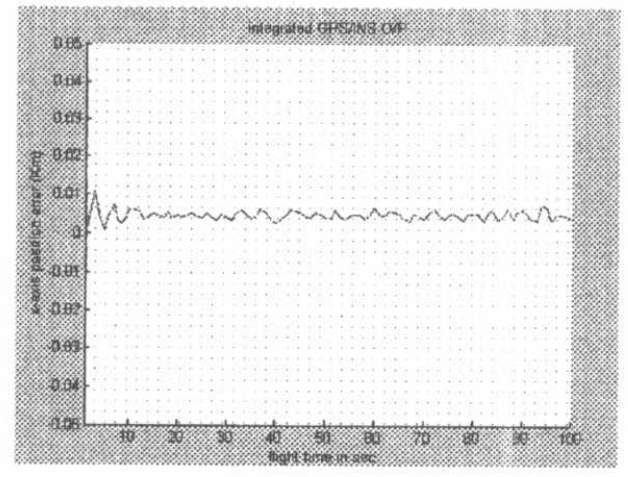

Fig. (6-a) Error in position in $\mathrm{X}$-axis

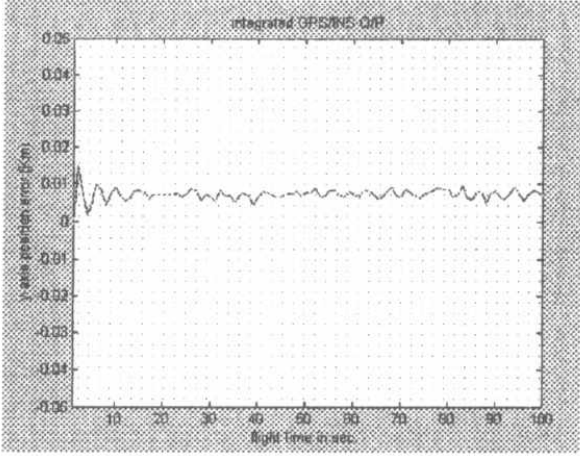

Fig. (6-b) Error in position in Y-axis 


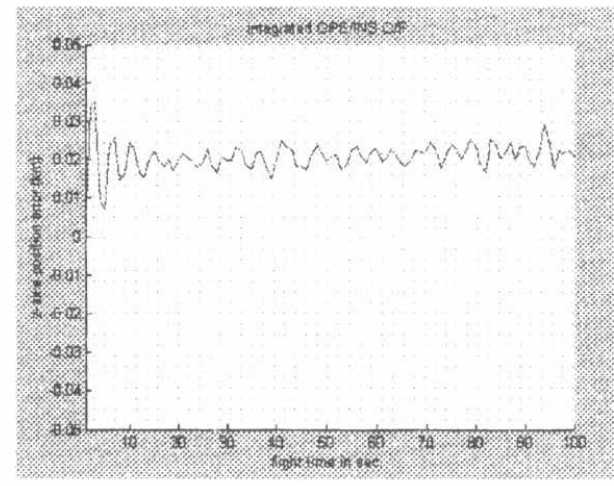

Fig. (6-c) Error in position in Z-axis

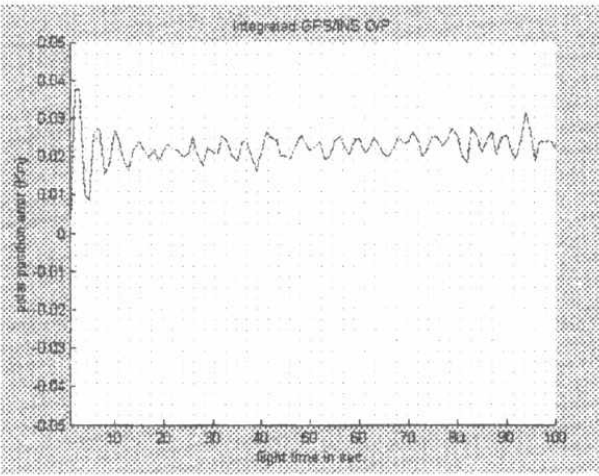

Fig. (7) Error in Range in XYZ-plane

\section{Conclusions:}

This paper discussed a continuous calibration and alignment scheme for inertial navigation system using Cascaded Kalman filter.

It is clear from the given plots and table (1) that, the errors of stand-alone INS is accurately calibrated using data output from GPS receiver. So, the integration of GPS/INS navigation system provides the following advantages leading to a high performance system:

1- Continuous calibration of INS (INS error reset) and in-flight alignment of INS using accurate GPS position fix are possible.

2- Continuous navigation output including attitudes even when the GPS signal is lost due to instantaneous and continuous output from INS.

3- Increasing jamming immunity even in high dynamics environment due to nonjammable system (INS).

Table (1) Navigation Parameters RMS errors.

\begin{tabular}{|c|c|c|c|c|}
\hline $\begin{array}{c}\text { Nav. Parameters } \\
\text { RMS errors }\end{array}$ & $\begin{array}{c}\text { Kalman filter } \\
\text { method }\end{array}$ & $\begin{array}{c}\text { Direct } \\
\text { method }\end{array}$ & INS stand-alone & $\begin{array}{c}\text { Integrated } \\
\text { GPS/INS }\end{array}$ \\
\hline$\Delta \mathbf{X}[\mathrm{m}]$ & 15 & 25 & 56 & 26 \\
\hline$\Delta \mathrm{Y}[\mathrm{m}]$ & 17 & 28 & 87 & 32 \\
\hline$\Delta Z[\mathrm{~m}]$ & 28 & 50 & 43 & 21 \\
\hline$\Delta \mathbf{V}_{\mathbf{x}}[\mathrm{m} / \mathrm{sec}]$ & 5 & 10 & 3.2 & 1.2 \\
\hline$\Delta \mathbf{V}_{\mathbf{y}}[\mathrm{m} / \mathrm{sec}]$ & 10 & 20 & 3.7 & 1.1 \\
\hline$\Delta \mathbf{V}_{z}[\mathrm{~m} / \mathrm{sec}]$ & 15 & 25 & 2.8 & 1.9 \\
\hline$\Delta \mathbf{R}[\mathrm{m}]$ & 36.03 & 62.3 & 53 & 34 \\
\hline
\end{tabular}




\section{Reference:}

[1] Brown, G.R.,"Introduction To Random Signal Analysis And Filtering": John Wiley \& Sons 1992.

[2] D.J.Dailey \& B.M.Bell,"A Method for GPS Positioning":[EEE Trans.on AES.Vol. No.5, pp 1148-1154,July 1996.

[3] Wade.H.F."Position-Location Solutions by Taylor-Series estimation": IEEE Trans.on AES.Vol. No2, pp 187-193,March 1976.

[4] K.V.Ramachandra \& V.S.Srinivasan,"Steady State Results For X, Y, Z Kalman Tracking Filter ":IEEE Trans.on. AES.pp 419-423,July 1977.

[5] K.V.Ramachandra "Optimum Steady-State Position, Velocity, and Acceleration Estimation using Noisy Sampled Position Data":IEEE Trans.on AES. , Vol. No.5,pp 705-708,September 1987

[6] Bernard Friendland," Optimum Steady-State Position, Velocity, and Acceleration Estimation using Noisy Sampled Position Data":IEEE Trans.on AES.,Vol. No6,pp 906-911,November 1973.

[7] Siouris, G.M.,"Aerospace avionics Systems": Acadimic Press 1993.

[8] D.H. Titterton \& G.L.Weston,"Strapdown Inertial Navigation Technology": Lavenham press 1998.

[9] Kenneth A.M,"Simulation Results For an Integrated GPS/Inertial Aircraft Navigation System": NAECON'76 Record, pp 841-848,1976.

[10] M. Napier," Integration of Satellite and Inertial Positioning Systems": Journal of the Institute of Navigation, vol. No. 1,pp 48-57, May 1990. 